\title{
Base, Holding, Initiation and Ending: A Four Stage Model of Brief Dynamic Psychotherapy Based on the Re-capitulation of Infantile Developmental Stages
}

\section{Alistair D Sweet ${ }^{\star}$}

School of Psychology, Queen's University, Belfast, Northern Ireland, UK

\begin{abstract}
This paper considers some of the ubiquitous elements of brief dynamic psychotherapy and suggests that these can be conceptualized within a four stage treatment model. The author suggests that distinct stages in the therapeutic relationship - base, holding, initiation/uncertainty and ending - can be discerned in the unfolding of the therapeutic relationship. A parallel is drawn with earliest developmental processes in the life of the individual and the four stage model is situated in relation to these earliest developmental vicissitudes.
\end{abstract}

Keywords: Stages; Model; Base; Holding; Initiation; Uncertainty; Ending

\section{Introduction}

The core focus in short-term psychodynamic psychotherapy is on the relationship between the therapist and the patient and the ways in which this relationship encapsulates a repetition of the core developmental conflicts that have led to the evolution of psychopathological symptoms and the global personality structure [1-4]. Addictive behaviours, for instance, are viewed as an attempted compromise solution to the individual's core conflicts and are consistent with a self-medication model of addiction [5]. What I term here attachment base exploratory psychotherapy should be utilized only after a period of stabilization, in essence abstinence from addictive behaviours, has been achieved. Generally this abstinence should be ongoing for three months plus. Individuals can be assisted in achieving such abstinence through behavioural supportive therapy and through involvement with self-help twelve step programmes.

The model of brief dynamic psychotherapy considered and presented here is based on a four stage model of psychotherapy which posits a de-regulation in the attachment pattern [6,7] and in the internalization of metalizing capacities (Bateman and Fonagy, 2001), as critically influencing later patterns of adult psychopathology $[4,8]$.

\section{The Four Stage Model}

Singular psychotherapeutic approaches often appear doctrinaire and so rigidly cast that many practitioners have explored integrative techniques, some clinicians now referring to themselves as integrative psychotherapists/counselors. Is it practical, or indeed possible, to distil certain key elements of the therapeutic process that appear to apply and to emerge universally in clinical practice, whether such practice is based on psychodynamic, cognitive, behavioural, or personcentered principles? Might it be that psychotherapeutic engagement unfolds primarily not on the basis of the clinician's orientation or theoretical application but rather on the sequential needs common to each presenting individual as these are related to the earliest stages of development and subsequently replayed in the therapeutic relationship? Here I suggest that four key stages appear to occur, in the therapeutic process: 1. Base, 2. Holding, 3. Uncertainty/Initiation and 4. Ending. The hypothesis advanced is that these stages will unfold irrespective of the presenting issues of the individual, or of the particular theoretical orientation of the practitioner. Whilst time-limited psychotherapy, based on this model, can be extremely helpful for particular patient/ client groups, e.g. circumscribed phobias, moderate levels of depressive anxiety, and adjustment disorders, other individuals will require longer term treatment. In both groups, whether short or longer- term treatment is undertaken, it is suggested that the key stages will unfold according to the patient's intra and inter-psychological timescale, not on the basis of the therapist's.

The psycho-physiological prototypes for the stages of base, holding, initiation/uncertainty and ending are considered to be: the womb, paternal matrix, acquisition of locomotion and individuation. It is suggested that the stages of treatment reify these early developmental milestones in the life of the individual. On this basis we can consider the re-deployment by individuals, during the treatment process, of their earliest object and self representations, mirroring their adaptation to the prevailing conditions of the infantile base, holding, loco-motor and individuating environments. Development is not conceived to unfold on a purely linear basis, either during infancy or in the psychotherapeutic relationship. Rather it is often the case that individuals evidence vacillation, for instance in wishing to retreat from the initiation/uncertainty stage to the holding stage or indeed from the holding stage to the base stage. There follows a brief description of how the 4 stage model can be conceptualized and applied in timelimited psychotherapeutic interventions. It should be borne in mind that the model is in no sense prescriptive; rather it is presented here to contextualize aspects of the 4 stages as these often tend to appear in treatment settings. For the purposes of illustration the following is based on 16 individual weekly sessions, of fifty minutes duration.

\section{Sessions 1-4: Base}

The primary focus, both consciously and unconsciously, during the initial treatment sessions is the creation of a secure base from which development can proceed. Pivotal in the creation of such a base is the manner in which the assessment interview is conducted. This will

Corresponding author: Alistair D Sweet, School of Psychology, Queen's University, Belfast, Northern Ireland, 40 Elmwood Avenue, Belfast, BT9 6AZ, Northern Ireland, United Kingdom, E-mail: alistairdsweet@yahoo.co.uk

Received December 18, 2012; Accepted December 19, 2012; Published December 28, 2012

Citation: Sweet AD (2013) Base, Holding, Initiation and Ending: A Four Stage Model of Brief Dynamic Psychotherapy Based on the Re-capitulation of Infantile Developmental Stages. J Psychol Abnorm Child 1: e101. doi:10.4172/23299525.1000e101

Copyright: $(2013$ Sweet AD. This is an open-access article distributed under the terms of the Creative Commons Attribution License, which permits unrestricted use, distribution, and reproduction in any medium, provided the original author and source are credited. 
normally be carried out over at least ninety minutes, though may on occasions, last up to two hours.

A semi-structured assessment interview format can enable the therapist and patient to illuminate key developmental factors that may have precipitated presenting issues. More recent and current life circumstances, for instance loss of employment, bereavement, separation from a partner, should also be recorded and the emotional impact on the patient assessed. During the assessment interview the clinician should also be able to gain an impression of the patient's personality structure, recognizing optimal levels of functioning and performance and measuring these against impingements and their affects. In the vast majority of patients a detailed life history, taken in a considerate and sensitive manner, encourages the foundation of a secure therapeutic base from which clinical treatment can proceed. Upon completion of the assessment interview the therapist and patient, working collaboratively, should be in a position to agree the provisional goals of treatment. Some patients in these early sessions will work more effectively with relatively scheduled and directed approaches (e.g. cognitive or cognitive behavioural interventions), whilst others will require a non-interventionist/analytical mode of working. Careful attention by the therapist to the patient's primary need, the facilitation of the secure base, is a pre-requisite in establishing and fostering a viable working alliance.

The suggestions made here challenge the exclusive efficacy claimed by certain proponents of various psychotherapeutic orientations. It is suggested that rather than applying "wholesale" a particular form of psychotherapy or counseling, the patient should lead the clinician; the latter being attentive to the former's individual needs. On this basis it is suggested that therapists can benefit greatly by having a working knowledge, based on a sound theoretical understanding, of the main forms of psychotherapy and counseling, such as; psychodynamic, cognitive, cognitive-behavioural, person centered and attachment based approaches.

\section{Sessions 5 to 8: The holding environment}

Following the initial phase of treatment, when a secure base has been established, the holding stage [9] is entered. This second stage of treatment can be conceptualized as the provision of a holding environment in which thoughts, feelings and emotions can be explored. For patients/clients this phase presents a necessary opportunity to test the capacities and limits of the therapeutic frame. In other words patients need to assure themselves that they can be safely and securely held. Holding within the therapist/patient relationship will be experienced as impinging when directiveness/boundary enforcement is applied in a restrictive and thoughtless manner. Conversely, when the therapist is lax or inattentive the client experiences a sense of abjection and neglect. The maintenance of a fluid and adaptive holding environment, which is dictated by the imminent needs of the patient, encourages a sense of exploratory flexibility and movement.

Interpretation and challenge, when provided constructively by the therapist/counselor, can be met with a range of responses by individuals. However it should be borne in mind those verbalisations that have typically been considered to indicate resistance, such as "if", "but", "when" etc., and instances of acting out, will often emerge during this stage. For many individuals such modes of verbal and behavioural action may have, in the past, been the only viable ways of managing intense, and potentially overwhelming psychological pain. The therapist's recognition of this, and the ability to communicate and encourage such recognition to the patient, is central to the therapeutic work of the holding stage.

\section{Sessions 9-12: Uncertainty and initiation}

Uncertainty and initiation are intimately related. Just as the young infant takes faltering steps, with uncertainty as to the outcome of their inchoate locomotion, so to do individuals who attempt new modes of communicative behaviour, both verbal and non-verbal. Initiation can manifest itself in many ways, often with patients/clients becoming more assertive, both with their therapist/counselor and with others. Such increasing assertiveness appears to rest on a developing sense of being able to contain thoughts, feelings and emotions that were previously experienced as being frightening, terrifying or potentially overwhelming. Physical changes are often seen in patients/clients during this stage of treatment. These are commonly reflected in gesture, posture, facial expressions and dress mode. Playing is the central theme of the uncertainty/initiation stage, with a key realization based on the acceptance of uncertainty, and that chaos simply is chaos, whether it is organized or not.

\section{Sessions 13-16: Ending}

Endings recapitulate loss. The central theme in the closing stages of brief treatment involves the recognition and acceptance of ending and loss. What appears of primary importance is the realization that therapeutic work involves a period of quite rapid change, with these changes being reflected in the range and intensity of emotional states experienced by the patient. When therapy ends the therapeutic process does not, as many of the issues raised during treatment continue to be worked through, both consciously and unconsciously, following the formal end of regular appointments. As in the preceding stage, uncertainty and initiation, some patients must progressively, and more slowly, come to terms with separation and ending. Telephone calls and letters may be received, or the patient may come in person once more to see the therapist/counselor. Rigidity in not acknowledging such individual needs, by avoidance on behalf of the practitioner, for instance, may indicate unresolved aspects of the therapist's relationship with the client/patient, rather than particular problems in the latter. Each of us, in our own time, attempts to come to an acceptance of ending and loss, and the nature of this individual process should I think be respected.

\section{Setting the 4 Stage Model in Context}

The model here presented, as the reader will note, is based on a pattern of environmental adaptation. Adaptation, so conceived, involves a process of physical and psychological change in relation to the evolving habitus of the individual. It is suggested that the therapeutic frame and the person of the therapist/counselor represent a transposed version of the early infantile environment, in which self/object relations were internalized. Dominant adult personality structures, whether these are psychotic, borderline or neurotic, can be located within the 4 stages. Those individuals presenting with psychotic personalities appear to evidence conflicts indicative of developmental arrest at the base/holding stages. Borderline and narcissistic pathology primarily indicate conflicts at the holding/uncertainty-initiation boundary. Neurotic patients struggle with uncertainty/initiation and ending. It is suggested that underscoring the psychological conflicts and pathological forms of behaviour that emerge in such conditions there are to be found distortions of the primary attachment relationship [10] Such distortions may be relatively mild to moderate, or they may be gross and chaotic. Following $[11,12]$ such impingements in earliest object relations can be clinically evaluated, in terms of triadic intrapsychic representations, which underpin psychological disturbance and the behaviours that ensue. These internalized triadic structures 
involve dominant self, object and affective constellations, of an infantile origin, which are subsequently re-activated and re-deployed in specific situations and under certain conditions.

Time-limited and brief psychotherapy appears most efficacious in assisting individuals to become more aware of core conflicts, and subsequently to initiate change, when internalized self, object and affective representations are illuminated through construction and interpretation of the transference.

\section{Conclusion}

In this brief paper I have advanced the idea that regardless of the particular type of psychotherapeutic treatment that is offered the patient there are therapeutic constants that emerge again and again in the relationship between the clinician and the patient. It is suggested that distinct stages can be discerned though these may not always unfold in a predictably linear way. The 4 stages highlighted here - base, holding, uncertainty/initiation and ending - are conceptualized to mirror psycho-physiological developmental processes that have long been understood to underscore separation and individuation during early childhood. It has been suggested that an awareness of these unfolding stages in the therapeutic relationship can assist clinicians in gaining a better understanding of the individual needs of the patient. This can facilitate flexibility in creating a therapeutic environment that is good enough in terms of enabling and encouraging psychological growth and development, even over relatively brief periods of psychotherapeutic contact.

\section{References}

1. Malan D (1992) The most important development since the discovery of the unconscious. In: EH Davanloo (Edr) Short-term Dynamic Psychotherapy. Aronson, Northvale, New Jersey and London.

2. Davanloo H (1996) A method of short-Term dynamic psychotherapy. In: Shortterm dynamic psychotherapy. Jason Aronson, Northvale, New Jersey and London.

3. Blatt S, Diamond D (2000) Attachment Research and Psychoanalysis Theoretical considerations Part 1. Routledge, New York and London.

4. Sweet AD (2011) Elements of psychotherapeutic assessment and treatment with structured and under-structured personalities. British Journal of Psychotherapy 27: 4-18.

5. Khantzian EJ (1985) The self-medication hypothesis of addictive disorders: focus on heroin and cocaine dependence. Am J Psychiatry 142: 1259-1264.

6. Bowlby J (1979) The making and breaking of affectional bonds. Routledge, New York and London.

7. Bowlby J (1988) A secure base: Clinical applications of attachment theory Routledge, London.

8. Sweet AD (2010) Automata states and their relation to primitive mechanisms of defence. Psychoanalytic Psychotherapy 24: 101-114.

9. Winnicott DW (1965) Maturational processes and the facilitating environment Studies in the theory of the emotional development. Hogarth Press, London.

10. Fonagy $P$ (2001) Attachment theory and psychoanalysis. Other Press, New York.

11. Bowlby J (1969) Attachment and Loss: Separation: anxiety and anger. Basic Books, New York.

12. Kernberg OF (2004) Contemporary controversies in psychoanalytic theory technique, and their applications. Yale University Press, New York. 